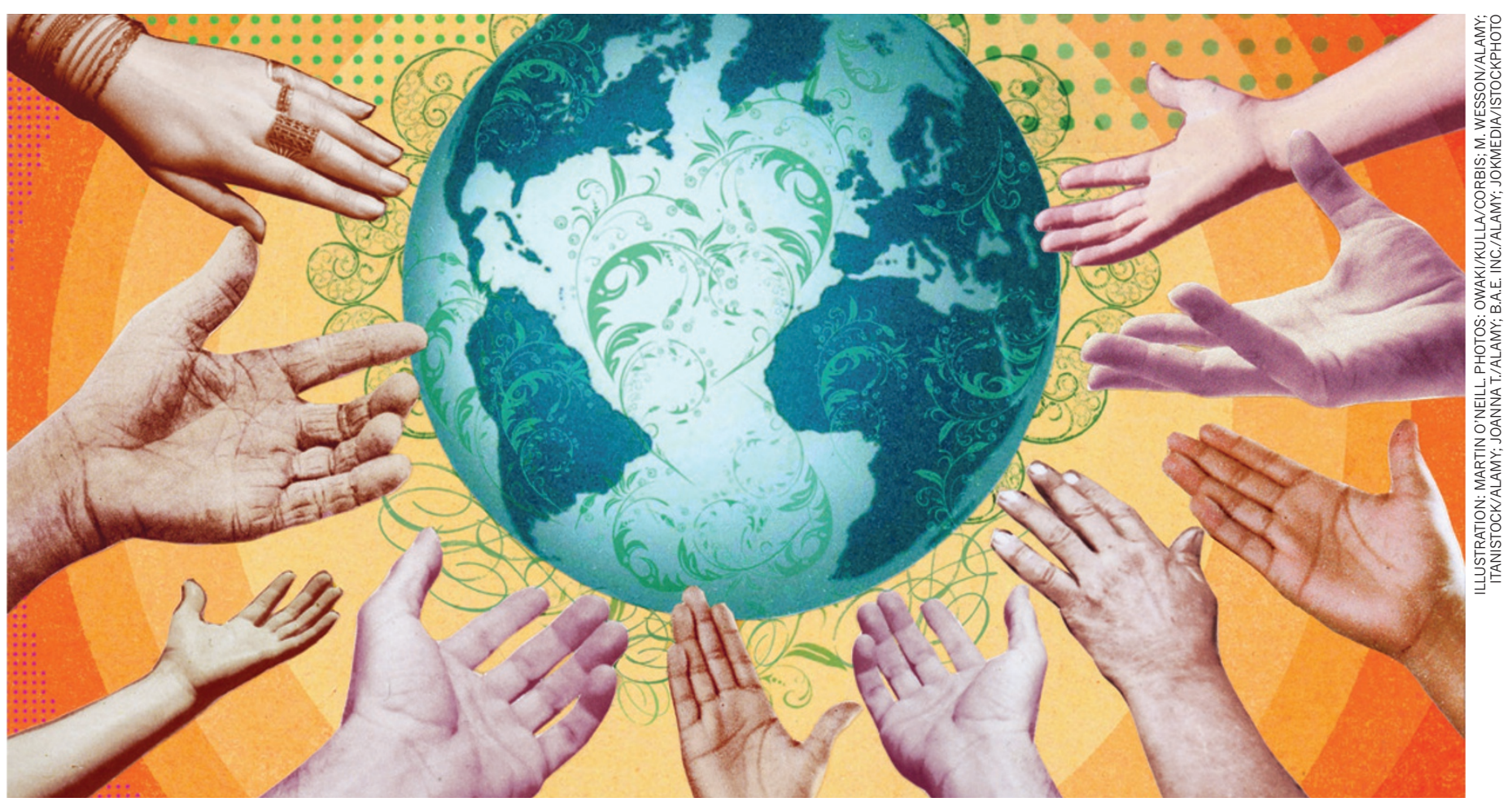

\title{
Listen to the voices of experience
}

\section{The intergovernmental body for biodiversity must draw on a much broader range of knowledge and stakeholders than the IPCC, say Esther Turnhout and colleagues.}

$\mathrm{I}$ $\mathrm{n}$ April this year, the Intergovernmental Platform on Biodiversity and Ecosystem Services (IPBES) was born in Panama City. It hopes to gain similar global scientific authority and policy influence for biodiversity to what the Intergovernmental Panel on Climate Change (IPCC) has for climate change - although not the same opposition and controversies.

Decisions have been made about the platform's functions and guiding principles. But many important issues regarding funding, expertise and institutional design are yet to be resolved. The interim secretariat has invited input into the drafting of a work programme as well as nominations for a multidisciplinary expert panel.

The IPBES's next plenary meeting is in early 2013. A window of opportunity therefore remains for it to learn from more than 20 years of IPCC experience, to better marry legitimacy and effectiveness. Here's how.

The IPCC focused on producing standardized assessments, with one view of what counts as relevant and valid knowledge for climate change: peer-reviewed science. This approach overshadowed arguably more important tasks: synthesizing wider perspectives about changing climates and spurring action by multiple policy actors.

The IPBES must not adopt such tunnel vision. Simply generating and communicating scientific knowledge is not sufficient to combat biodiversity loss. Current IPBES documentation, such as the provisional work programme and technical background documents, suggests that the platform aims to serve as a clearing house that guarantees the global availability of all biodiversity knowledge that has been standardized and scientifically validated.

This IPCC-like focus might be attractive to 'elite actors', from natural scientists to national governments, but it omits many other important stakeholders and knowledge-holders, including indigenous people, businesses, farmers, community partnerships and fishers. What counts as legitimate knowledge, and how it is generated, influences its practical effectiveness.

The knowledge of traditional and 'ordinary' citizens might not meet scientific criteria or be amenable to standardization, but ignoring or misappropriating such experience, undermines the possibilities for innovation ${ }^{1}$. For example, Jan Douwe van der Ploeg, an anthropologist at Wageningen University in the Netherlands, has shown how the agricultural revolution endangered the livelihoods of Andean hill farmers when the miracle crops did not deliver, and de-skilled them because it over-rode their local knowledge, which had led to sustainable yields for generations ${ }^{2}$.

\section{MEETING OF MINDS}

Scientific and experience-based knowledge can come together. A good example is an initiative by the Natural History Museum in London in which fly-fishers' expertise has made official water-monitoring schemes more realistic and robust ${ }^{3}$. Another example is from India, where incorporating local 
knowledge in scientific censuses improved tiger management ${ }^{4}$.

Admittedly, different types of information require different processes of validation. So the IPBES expert panel must design and use appropriate review protocols. These could involve the recruitment of multiple peer communities, including specialist citizens, biodiversity practitioners and place-based experts, to assess the credibility, quality and practical relevance of knowledge.

An important step in this direction is the development of experimental partnerships with established and trusted organizations such as natural history museums, zoos and botanical gardens. Many of these organizations are becoming places that bring together research and public engagement, often through citizen science. The Berlin Museum for Natural History was recently awarded a grant by the German Federal Cultural Foundation to work on the role of natural history museums in IPBES and in global biodiversity governance. At the conference of the parties to the UN Convention on Biological Diversity in Nagoya, Japan, in 2010, such organizations were mentioned as important nexus of practical knowledge and local action through which biodiversity awareness could be heightened.

\section{CHECK THE LABEL}

There is no single scientific definition of biodiversity ${ }^{5}$, nor is there one that does justice to the many ways of living with and knowing nature that human cultures have developed. The IPBES has not taken adequate notice of this and is promoting a predominantly science-based understanding of biodiversity, with ecosystem services taking centre stage.

This focus reduces biodiversity to an object of exploitation and runs the risk of bringing it even further into a system of market exchange ${ }^{6}$. Although the concept of ecosystem services prompts private-sector and governmental responses in the developed world, it alienates important political actors. Objectors include Bolivian, Ecuadorian and Cuban delegates at IPBES meetings, and other developing countries at the Convention on Biological Diversity. Moreover, scientists from several disciplines question the benefits of the ecosystem-services approach ${ }^{7}$.

We ask that the IPBES respect the manifold meanings biodiversity has for people. Monetary, aesthetic and sacred values should be given equal prominence in policy discussions of what biodiversity and ecosystems offer to humans, for example.

The IPCC claims to be both policy-relevant and policy-neutral. But the IPCC's knowledge is not equally relevant for all actors, so it cannot claim to be entirely neutral. Different policy-makers want and need different things, so any one framing of a problem - be it scientific, economic or ethical - signals who will act and how ${ }^{8}$. For example, by promoting 'global temperature' as the standardized unit to express the problem of global-warming, the IPCC deems only certain types of action relevant, whether it be mitigating climate change or manipulating the stratosphere. Such standardization is good for modellers and funders, but it has failed to inform effective, diverse and local adaptation and mitigation policies and practices.

Another reason why climate policies have been hard to enact is the IPCC's implicit assumption that the key actors will assent to top-down knowledge and that national and global institutions are synonymous with 'the policy world'. Legislation is essential, but for global issues such as climate and biodiversity

\section{BIODIVERSITY}

\section{Rules of engagement for the IPBES}

1. Operate not as a centralized global organization, but as global coordinator of a distributed network that can be sensitive to local knowledge, needs and conditions. 2. Address all mandated functions simultaneously and in a balanced way so that non-elite actors are not placed in an end-of-pipe position.

3. Facilitate broad discussion of the terms and methodologies used to define, understand, assess and conserve biodiversity; and be explicit about contested assumptions.

4. Ensure diverse representation in activities and decisions. Expert panel should include natural scientists, social scientists, humanities researchers, biodiversity practitioners and indigenous-knowledge networks, with accreditation criteria and selection processes made public.

5. Experiment with ways to validate and maintain quality control, such as sensible narratives and citizen panels.

6. Embrace dissenting views and perspectives to build trust among represented parties - for example, through minority reporting instead of pursuing consensus.

7. Work with trusted civic organizations and networks at the interface of science, citizens, business and culture.

8. Have rolling and overlapping timetables for different products, rather than delivering a single 'big-bang report' every six years. 9. Reflect regularly to identify areas for improvement. it is not sufficient ${ }^{9}$.

Ending practices that destroy biodiversity - such as uncontrolled mono-crop agriculture or large-scale deforestation - requires diverse and locally appropriate actions. The IPBES must therefore forge productive and trusted connections between organized global knowledge and the many biodiversity actors operating at multiple levels and scales. It should draw inspiration from existing research-practice networks, such as community forestry partnerships and the Agreco network developed by local agriculturalists in Santa Catarina, Brazil. Working with the Federal University of Santa Catarina, Agreco transformed its chemicals-intensive tobacco farms into a more biodiverse and multifunctional agricultural economy and society and now also produces grains, fruits and honey ${ }^{10}$.

\section{STEPS TO SUCCESS}

The IPBES will be deemed successful if it manages to increase the available range of policy interventions, based on a broad set of relevant ecological knowledge, to slow biodiversity loss at all scales. We believe that the IPBES can achieve this and need not become just another remote and disconnected international body, if it follows our nine recommendations (see 'Rules of engagement for the IPBES'). Although perhaps uncomfortable and unpredictable, the open and experimental approach we outline is necessary for meeting the momentous challenge of biodiversity loss.

Esther Turnhout is in the Forest and Nature Conservation Policy Group, Wageningen University, the Netherlands. Bob Bloomfield is at Science in Society, The Natural History Museum, London, UK. Mike Hulme is in the Science, Society and Sustainability Group, School of Environmental Sciences, University of East Anglia, Norwich, UK. Johannes Vogel is director-general of the Museum für Naturkunde, Berlin, Germany. Brian Wynne is at the UK ESRC Centre for Economic and Social Aspects of Genomics, Cesagen, Lancaster University, UK. e-mail:esther.turnhout@wur.nl

1. Agrawal, A. Dev. Change 26, 413-439 (1995).

2. van der Ploeg, J. D. in An Anthropological Critique of Development: The Growth of Ignorance (ed. Hobart, M.) 209-227 (Routledge, 1993).

3. Waterton, C. Salmo Trutta 6, 56-58 (2003).

4. Karanth, K. U. et al. Anim. Conserv. 6, 141-146 (2003).

5. Royal Society Measuring biodiversity for conservation (Royal Society, 2003).

6. Hayden, C. When Nature Goes Public: The Making and Unmaking of Bioprospecting in Mexico (Princeton Univ. Press, 2003).

7. Walker, S. et al. Conserv. Lett. 2, 149-157 (2009).

8. Wynne, B. in Environmental Threats: Perception, Analysis and Management (ed. Brown, J.) 233-247 (Frances Pinter, 1989).

9. William, W. B. lowa Law Rev. 89, 3-53 (2003).

10.Pinheiro, S. in The Earthscan Reader in Sustainable Agriculture (ed. Pretty J.) 352-361 (Earthscan, 2009). 